\title{
DOA measurements using synthetic aperture method
}

\author{
Kazuma Tomimoto ${ }^{\text {a) }}$ and Ryo Yamaguchi \\ $R \& D$ Division, Softbank Corp., Telecom Center Building East Tower 13F, \\ 2-5-10 Aomi, Koto-ku, Tokyo 135-0064, Japan
}

a)kazuma.tomimoto@g.softbank.co.jp

Abstract: It is important to understand the DOA characteristics in order to develop massive-array antennas for $5^{\text {th }}$ generation mobile communication systems. We introduce a simple but effective method for measuring direction of arrival (DOA) that combines synthetic aperture (SA) processing with a receiving-system that consists of a turntable, vector receiver, and horn antenna. This report shows by simulation the effectiveness of synthetic aperture processing. The validity of this method is clarified by measurements in a chamber.

Keywords: synthetic aperture (SA), direction of arrival (DOA) measurement, antenna, propagation

Classification: Antennas and Propagation

\section{References}

[1] ARIB 2020 and Beyond Ad Hoc Group, White paper "Mobile communications Systems for 2020 and beyond," October, 2014, http://www.arib.or.jp/english/ 20bah-wp-100.pdf.

[2] N. Tran, T. Imai, and Y. Okumura, "Measurement of indoor channel characteristics at $20 \mathrm{GHz}$ band," International Symposium Antennas and Propagation (ISAP), 2015.

[3] R. Schmidt, "Multiple emitter location and signal parameter estimation," IEEE Trans. Antennas Propag., vol. 34, no. 3, pp. 276-280, March 1986. DOI:10. 1109/TAP.1986.1143830

[4] B. D. Rao and K. V. S. Hari, "Performance analysis of Root-MUSIC," IEEE Trans. Acoust. Speech Signal Process., vol. 37, no. 12, pp. 1939-1949, 1989. DOI:10.1109/29.45540

[5] R. Roy and T. Kailath, "ESPRIT-estimation of signal parameters via rotational invariance techniques," IEEE Trans. Antennas Propag., vol. 37, no. 7, pp. 984-995, July 1989. DOI:10.1109/29.32276

[6] R. Yamaguchi, Y. Kimura, K. Komiya, and K. Cho, "A far-field measurement method for large size antenna by using synthetic aperture antenna," Proc. of EuCAP2011. 


\section{Introduction}

Studies on $5^{\text {th }}$ generation mobile communication systems using high frequency bands, $6 \mathrm{GHz}$ or above, are active [1]. In order to solve the problem of the high propagation loss, massive-array antennas such as Massive MIMO are attracting attention. Develop such an antenna demands an understanding of the propagation characteristics as the antennas have narrow beam characteristics. Therefore, a number of studies have described direction of arrival (DOA) measurements. There are 2 approaches to DOA measurements. The first uses a large scale aperture antenna such as a parabolic antenna or horn antenna with channel sounding system [2]. However, the antenna and channel sounding system must be redesigned when the measurement frequency is changed. The second uses just a few antenna elements with a high resolution estimation algorithm such as MUSIC, ESPRIT $[3,4,5]$. However, this demands calibration between each antenna. We combine the synthetic aperture technique [6] with a receiving-system consisting of a turntable, vector receiver, and horn antenna, to achieve higher angular resolution than the former approach and easier measurements than the latter. Amplitude and phase characteristics are obtained within the range of -180 degrees to 180 degrees as the receiving antenna is placed on a turntable $R$ [cm] from the center, and obtained data is subjected to off-line synthetic aperture (SA) processing. Hence, the proposed method offers DOA measurements with high angular resolution by constructing a virtual circular arc antenna. It is possible to use a broadband receiving antenna without preparing an array antenna for each frequency, so this proposal offers wideband propagation characteristics and high versatility. In this report, we confirm the effectiveness of SA processing by simulation. In addition, its usefulness is verified by experiments in a chamber.

\section{Measurement principle and measurement parameters}

The proposal improves the DOA angular resolution by constructing a virtual circular arc synthetic aperture array antenna with radius of $R$. Assuming that the wave source (Tx) lies in the far-field, the measurement principle of this method is shown in Fig. 1(a). The receiver antenna ( $\mathrm{Rx})$ is placed $R[\mathrm{~cm}]$ from the center of the turntable, and the transmission characteristics between receiver antenna $(\mathrm{Rx})$ and wave source (Tx) are measured while the turntable is rotated from -180 degrees to 180 degrees. Array weights corresponding to the optical path difference are needed for constructing the virtual circular arc array antenna. The synthetic aperture array antenna is formed according to (1) by using measured electric fields and array weights.

$$
E_{S A}\left(\theta_{i}\right)=\sum_{j=-N}^{N} E\left(\theta_{i}+\phi_{j}\right) \cdot \exp \left\{j k R\left(1-\cos \left(\phi_{j}\right)\right)\right\}
$$

$E_{S A}\left(\phi_{j}\right), E\left(\theta_{i}+\phi_{j}\right), \phi_{j}, \theta_{i}$ and $\exp \left\{j k R\left(1-\cos \left(\phi_{j}\right)\right)\right\}$ are the synthesized electric field, the measured electric field, the setting angle of the $j$ 'th virtual antenna, the rotation angle, and the array weight, respectively. $2 N+1$ receiver antennas $(\mathrm{Rx})$ are formed at intervals of one degree. $2 N+1 \mathrm{Rx}$ at $\theta_{i}=0$ degrees is the real receiver antenna, $2 N$ of $2 N+1$ is the virtual antenna. The response from $\mathrm{Rx}$ is 
captured by the vector receiver, and then synthesized numerically in a PC in an offline manner.

Simulation and measurement parameters are shown Fig. 1(b). The receiving antenna is a horn antenna. Radiation pattern of the horn antenna assumed in the simulation is given by eq. (2). It is set to match the $3 \mathrm{~dB}$ beam-width of the horn antenna used in actual measurements.

$$
E\left(\theta_{i}+\phi_{j}\right)=\frac{1+\cos \theta_{i}}{2} \cdot \frac{\cos \left(\frac{\pi}{2} \cdot 1.15 \lambda\right)}{1-(1.15 \lambda)^{2}} e^{-j k R\left(1-\cos \theta_{i}\right)}
$$

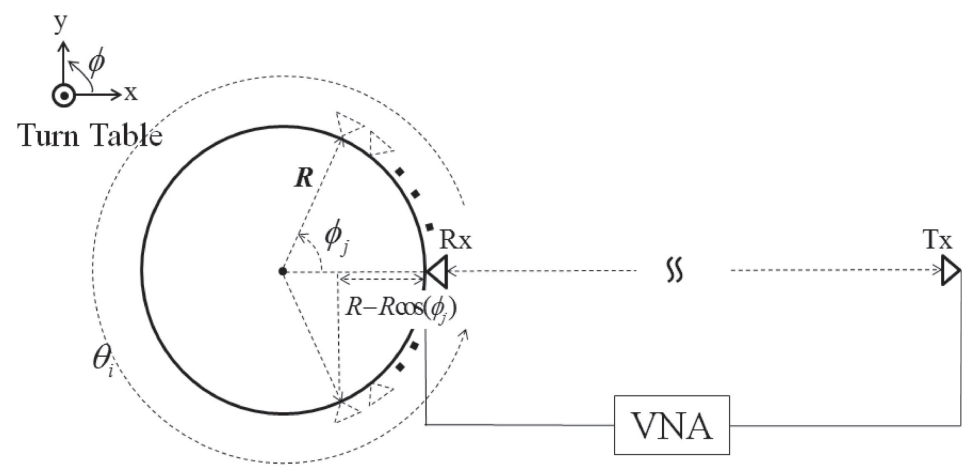

(a): Measurement Principle

(b): Measurement and simulation Parameters

\begin{tabular}{|c|c|}
\hline Frequency $[\mathrm{GHz}]$ & 12 \\
\hline Antenna Element $(\mathrm{Rx})$ & Horn antenna \\
\hline Radius of array $(R)[\mathrm{cm}]$ & $20(8 \lambda)$ \\
\hline Wave source(Tx) direction $[\mathrm{deg}]$. & 0 \\
\hline Angle step [deg.] & 1 \\
\hline Number of elements $(2 N+1)$ & 121 \\
\hline
\end{tabular}

Fig. 1. Principle and parameters

\section{DOA spectra by SA processing}

DOA spectra with and without SA processing are shown in Fig. 2(a). They are normalized against their respective peak values. In this paper, $3 \mathrm{~dB}$ beam-widths are defined as $3 \mathrm{~dB}$ resolution, $10 \mathrm{~dB}$ beam-widths are defined as $10 \mathrm{~dB}$ resolution. From Fig. 2(a), (b), the $3 \mathrm{~dB}$ resolution is 42.9 degrees without SA processing, and 4.9 degrees with SA processing. This result shows that the angular resolution can be greatly enhanced by acquiring the propagation characteristics given the radius from the center of the turntable and performing the SA processing offline. 


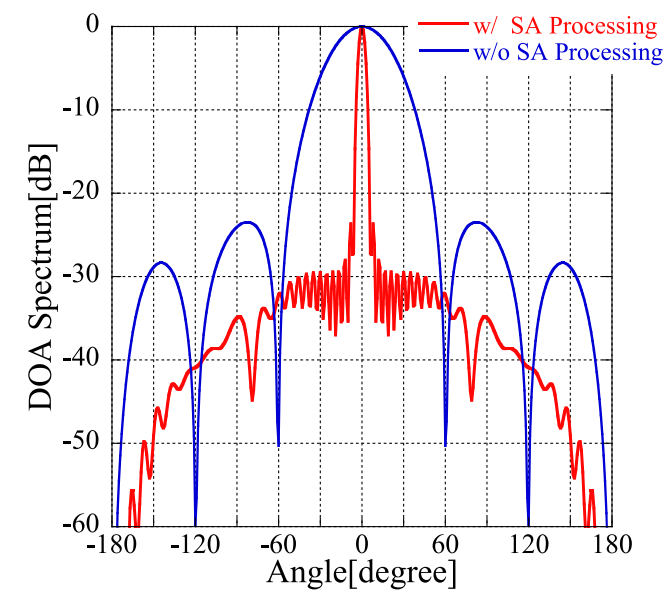

(a): DOA spectra

(b) Comparison in with and without SA processing

\begin{tabular}{|c|c|c|}
\hline & w/o SA processing & w/ SA processing \\
\hline $3 \mathrm{~dB}$ Res.[deg] & 42.9 & 4.9 \\
\hline 10 dB Res.[deg] & 76.2 & 8.5 \\
\hline
\end{tabular}

Fig. 2. Results of simulation

\section{Measurements in a chamber}

In order to confirm the usefulness of the proposed measurement method, we measure the DOA in chamber using a $12 \mathrm{GHz}$ band horn antenna. In accordance with the measurement principle, it SA processing is performed offline after obtaining the radiation characteristics. Fig. 3(a) shows the DOA spectra (measured and simulated) when one wave source is placed on the 0 degree axis. From Fig. 3(a), the $3 \mathrm{~dB}$ resolution is 4.9 degrees (simulated) and the 5.3 degrees (measured), which agree well. However, unwanted side-lobes lie on the -10 degrees direction. They indicate the influence of the amplitude and phase patterns of the antenna used in the measurements and the influence of the error between the phase center of the antenna and the rotation center. We think that it is necessary to apply a window function to reduce these side-lobes.

Wave sources were placed in the chamber at 0 degree and -10 degree directions, and DOA measurements of 2 waves were conducted. This technique makes it possible to know the arrival angle accurately. 


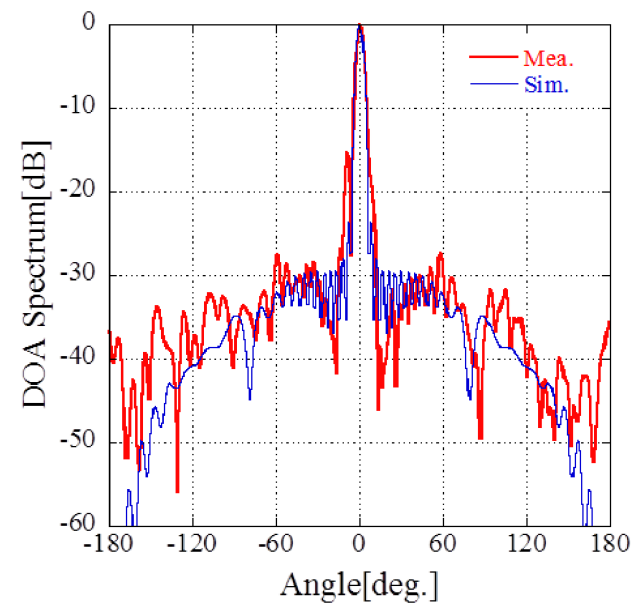

(a): DOA spectra for the case of one arrival wave

(b) Resolutions in Measurement

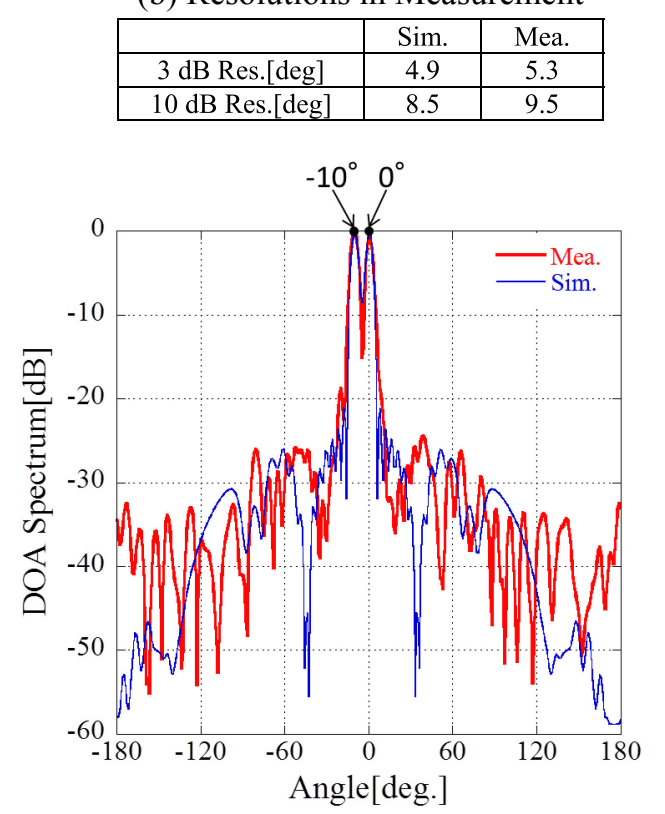

(c): DOA spectra for the case of two arrival waves

Fig. 3. Comparison of simulation and measurement results

\section{Conclusions}

In this paper, we introduced a DOA measurement method that uses a turntable, vector receiver, horn antenna and synthetic aperture processing; its usefulness was confirmed by simulation and measurements. This proposal greatly improves the angular resolution by forming a virtual array as the receiver set at a given radius processing the data offline. Furthermore, by using a broadband antenna, it has the merit that various frequencies can be measured without changing the measurement system. In the future, delay wave measurements will be realized by this measurement method. In addition, we will conduct measurements in multipath environments and examine the measurement parameters. 